\title{
Utilização de recurso tecnológico para estratificação de risco cardiovascular em usuários do programa hiperdia: relato de experiência
}

\author{
Use of technological resources for cardivascular risk stratification in users of the hiperdia \\ program: experience report
}
Utilización de recurso tecnológico para estratificación de riesgo cardivascular en usuarios del programa hiperdia: relato de experiencia

Lucas Miléo Teixeira ${ }^{1 *}$, Alice Garcia de Oliveira1, Débora Mylena Azevedo Rosa1, Isadora Pina Simão ${ }^{1}$, Giselle de Oliveira Souza ${ }^{1}$, Juliana de Kássia Alves de Sousa ${ }^{1}$, Larissa Castro de Souza ${ }^{1}$, Raiane Bacelar dos Anjos ${ }^{1}$, Rosimere de Freitas de Sousa ${ }^{1}$, Victória Leal Ferreira ${ }^{1}$, Yasmin Cristino Monteiro $^{1}$, Idehize Oliveira Furtado Lima ${ }^{1}$.

\section{RESUMO}

Objetivo: Descrever as experiências dos discentes de Enfermagem na Estratificação de risco cardiovascular. Relato de Experiência: Trata-se de um estudo descritivo do tipo relato de experiência, realizado nas aulas práticas do componente curricular "Enfermagem Comunitária II" em uma Unidade Estratégia da Família em Belém/PA, através da estratificação de risco cardiovascular dos usuários da unidade, utilizando um aplicativo criado e disponibilizado pelo Ministério da Saúde que usa os parâmetros da tabela Framingham. Foram analisados 282 prontuários de usuários do programa Hiperdia e, constatou-se que somente $32 \%$ estavam aptos para a estratificação. Através da estratificação de risco cardiovascular foi possível traçar um perfil dos usuários desse programa e, verificou-se o predomínio do sexo feminino no programa e que os homens apresentaram um maior risco de desenvolver alguma doença cardiovascular em 10 anos. Considerações Finais: Com a utilização do aplicativo para a estratificação de risco cardiovascular confirmou-se que com a aplicação de recursos tecnológicos, como os aplicativos móveis, há um maior fornecimento de autonomia aos profissionais de saúde, melhorando assim a assistência para os usuários da unidade.

Palavras-Chave: Enfermagem, Doenças Cardiovasculares, Risco Cardiovascular.

\begin{abstract}
Objective: To describe the experiences of nursing students in the stratification of cardiovascular risk. Experience Report: It is a descriptive study of the experience report type, accomplished in the practical classes of the curricular component "Community Nursing II" in a Family Health Strategy Unit in Belém / PA, through the cardiovascular risk stratification of the users of the unit, using an application created and made available by the Ministry of Health that uses the Framingham table parameters. Were analyzed 282 medical records of Hiperdia users and it was verified that only $32 \%$ were suitable for stratification. Through the stratification of cardiovascular risk, it was possible to draw profile of the users of this program and it was verified the predominance of females in the program as well as which men had a higher risk of developing cardiovascular disease in 10 years. Final Considerations: With the use of the created application for a stratification of cardiovascular risk it was confirmed that the application of technological resources, such as mobile applications, there is a greater supply of autonomy for health professionals, improving services for the users of the unit.
\end{abstract}

Keywords: Nursing, Cardiovascular Diseases, Cardiovascular Risk.

1 Universidade do Estado do Pará- UEPA. *E-mail: lucasmileoteix@gmail.com 


\section{RESUMEN}

Objetivos: Describir las experiencias de los alumnos de Enfermería en la Estratificación de riesgo cardiovascular. Relato de Experiencia: Se trata de un estudio descriptivo del tipo relato de experiencia, realizado en las clases prácticas del componente curricular "Enfermería Comunitaria II" en una Unidad Estrategia de la Familia em Belém/PA, a través de la estratificación de riesgo cardiovascular de los usuarios de la unidad, utilizando una aplicación creada y puesta a disposición por el Ministerio de Salud que utiliza los parámetros de la tabla Framingham. Se analizaron 282 prontuarios de usuarios del programa Hiperdia y, se constató que sólo el $32 \%$ era apto para la estratificación. A través de la estratificación de riesgo cardiovascular fue posible trazar un perfil de los usuarios de ese programa y, se verificó el predominio del sexo femenino en el programa y que los hombres presentaron un mayor riesgo de desarrollar alguna enfermedad cardiovascular en 10 años. Consideraciones finales: Con la utilización de la aplicación para la estratificación de riesgo cardiovascular se confirmó que con la aplicación de recursos tecnológicos, como las aplicaciones de móviles, hay un mayor suministro de autonomía a los profesionales de la salud, mejorando así la asistencia para los usuarios de la unidad.

Palabras clave: Enfermería, Enfermedades Cardiovasculares, Riesgo Cardiovascular.

\section{INTRODUÇÃO}

Em consonância com os princípios da Atenção Básica foi criado o Plano de Reorganização da Atenção à Hipertensão Arterial e Diabetes Mellitus onde foi instituído o programa HIPERDIA, com intuito de estabelecer a necessária vinculação do usuário ao serviço de saúde e assim fornecer o atendimento de qualidade necessário para esses usuários. O HIPERDIA permite a geração de informações para a distribuição, dispensação e aquisição de medicamentos de uma maneira regularizada sistemática para todos os pacientes nele cadastrados (CARVALHO FC, et al.,2018).

As doenças cardiovasculares são a principal causa de mortalidade no mundo, resultantes tanto do envelhecimento da população quanto das mudanças epidemiológicas relativas às doenças. É estimado que somente no ano de 2017 ocorreram cerca de 383.961 óbitos por doenças cardiovasculares no Brasil (ROTH GA, et al., 2015). Dentre essas enfermidades, está à hipertensão arterial sistêmica (HAS), condição clínica multifatorial associada a alterações funcionais, estruturais e metabólicas, com consequente aumento do risco de eventos cardiovasculares fatais e não fatais (MALACHIAS MV, 2016).

Todavia, esses quadros clínicos podem ser evitados, se detectados precocemente os fatores de riscos, os quais não se limitam, somente, a questões genéticas e hábitos de vida, mas a presença de doenças crônicas não transmissíveis, como hipertensão e diabetes, sinalizadores de extrema relevância para o risco cardiovascular (BRASIL, 2018).

Diante disso, tecnologias podem ser introduzidas como apoio à decisão no processo de atendimento (CRUZ; KIPPLER, 2018). Nessa perspectiva então, é importante que o enfermeiro esteja em constante processo de capacitação teórico-prática, aprendendo e pesquisando, conhecendo as novas tecnologias, identificando seus conceitos e seja capaz de integrar e aplicar os novos adventos tecnológicos ao processo de cuidar em saúde (RAMOS S, et al, 2014).

Uma ajuda para facilitar esse processo de cuidado em saúde é o Escore de Risco de Framingham (ERF), vale ressaltar que é uma das principais ferramentas para avaliação cardiovascular com uma projeção para 10 anos criada em 1988, onde foi utilizado os determinados fatores de risco: Idade, sexo, HDL (High Density Lipoproteins) ou colesterol total, pressão, diabetes e tabagismo. Esse escore foi modificado no ano de 2002 pelo Terceiro Relatório do Painel de Especialistas em Detecção, Avaliação e Tratamento de Colesterol no Sangue Elevado em Adultos - Painel de Tratamento de Adultos III (NCEP ATP III). Nesta, para 0 tratamento e avaliação da dislipidemia, foram realizadas uma serie de modificações quanto à inclusão de tratamento da hipertensão, eliminação de diabetes do algoritmo, pontuação para o tabagismo, preferência do colesterol total ao LDL-(Low Density Lipoproteins) e também seu ajuste de acordo com a idade (SERAFIN GS, et al, 2014).

REAS/EJCH | Vol. Sup.27 | e835 | DOI: https://doi.org/10.25248/reas.e835.2019 Página 2 de $\mathbf{6}$ 
Atualmente, na consulta de enfermagem, para a estratificação de risco cardiovascular, é recomendada a utilização do Escore de Framingham visando uma assistência de enfermagem aprimorada e auxiliando no processo de enfermagem, pois através desta estratificação há a possibilidade de estimar o risco de cada indivíduo sofrer uma doença arterial coronariana nos próximos dez anos (BRASIL, 2013).

Assim, a estratificação de risco é de extrema relevância para que a equipe de saúde organize ações a ser oferecida a cada grupo de risco nas populações adstritas a determinada Atenção Básica de Saúde, visando identificar os estratos de morbidades cardiovasculares dos usuários desse território (BRASIL, 2017).

Por conseguinte, o enfermeiro possui papel importante na realização da estratificação de risco, elaboração de planos de cuidados para as pessoas que possuem condições crônicas no território, bem como na estruturação de um sistema de informação clínica efetivo em relação às condições crônicas de todas as pessoas usuárias junto aos demais membros da equipe (BRASIL, 2017).

Dessa forma, devido a não realização da estratificação de risco e da existência de um alto número de pessoas hipertensas e diabéticas na Estratégia de Saúde da Família (ESF) em um bairro periférico de Belém/PA, este estudo busca relatar o processo de estratificação do risco cardiovascular (RCV), através do uso de um aplicativo que calcula o risco que um indivíduo tem de desenvolver alguma doença cardiovascular em um período de aproximadamente 10 anos.

\section{METÓdOS}

Trata-se de um estudo descritivo do tipo relato de experiência que descreve a vivência dos discentes do Curso de Enfermagem da Universidade do Estado do Pará, na estratificação do RCV em usuários de uma ESF em Belém/PA, no período das aulas práticas do componente curricular Enfermagem Comunitária II, utilizando um aplicativo (Calculadora de Risco Cardiovascular), criado e disponibilizado gratuitamente pelo Ministério da Saúde (Telessaúde RS) em parceria com a Universidade Federal do Rio Grande do Sul.

Este estudo teve como fundamentação metodológica a teoria da problematização, postulada por Berbel no ano de 1995, conhecido como "Arco de Maguerez", o qual se divide em cinco etapas, para melhor sistematização da ideia. São elas: Observação da realidade, Levantamento dos Pontos-Chave, Teorização, Propostas de solução e Retorno a realidade (BERBEL NAN, 1995).

Na primeira etapa do "Arco de Maguerez", observou-se a realidade vivenciada pelos usuários da ESF, em seguida foi realizado o levantamento dos pontos-chave, ou seja, os pontos mais relevantes e críticos encontrados na unidade, à qual deveriam ser destacados e trabalhados posteriormente, sendo assim, definiram-se as doenças cardiovasculares como a temática de maior relevância a ser trabalhada na unidade.

Após definida a temática, realizou-se a teorização, pesquisando na literatura a fim de estabelecer as propostas de solução para a problemática, portanto, foi determinado que a melhor forma de estratificar os riscos cardiovasculares dos usuários da ESF seria através da utilização de um aplicativo que utiliza os parâmetros da escala de Framingham para realizar tal estratificação. Por fim, houve o retorno a ESF para analisar os prontuários dos usuários de cinco microareas (MA03, MA05, MA06, MA07 e MA08) que integravam o programa Hiperdia e, estratificar aqueles que estivessem aptos para tal. Os prontuários habilitados foram demarcados com fitas de coloração vermelha para RCV alto, amarelo para RCV intermediário e verde para RCV baixo.

\section{RESULTADOS}

Dos 282 prontuários analisados, somente 91 (32\%) estavam adequados para o estudo, ou seja, 191 (68\%) prontuários foram eliminados da pesquisa, uma vez que não continham todas as informações necessárias para a realização do cálculo do RCV, como sexo, idade (não superior a 75 anos), colesterol total, colesterolHDL, pressão sistólica, informação sobre uso de medicamento anti-hipertensivo, diabetes e tabagismo.

De acordo com as informações colhidas dos prontuários aptos, podemos notar que a $72,5 \%$ eram mulheres com média de idade de 57,5 anos e 27,5\% eram homens com média de idade 59 anos. 
Comparando o índice de RCV entre homens e mulheres, verificou-se que $33,3 \%$ das mulheres apresentaram RCV alto, 27,3\% RCV intermediário e 39,9\% RCV baixo, já entre os homens $68 \%$ apresentaram RCV alto, 24\% RCV intermediário e 8\% RCV baixo (Tabela 1).

Tabela 1 - Distribuição dos valores de sexo em comparação com porcentagens de risco para doença cardiovascular.

\begin{tabular}{|c|c|c|c|c|c|c|c|c|}
\hline \multirow[t]{2}{*}{ SEXO } & \multicolumn{2}{|c|}{$\begin{array}{c}\mathbf{N}^{\circ} \mathrm{DE} \\
\text { USUÁRIOS }\end{array}$} & \multicolumn{2}{|c|}{ RCV ALTO } & \multicolumn{2}{|c|}{ RCV INTERMEDIÁRIO } & \multicolumn{2}{|c|}{ RCV BAIXO } \\
\hline & $\mathbf{N}$ & $\%$ & $\mathbf{N}$ & $\%$ & $\mathbf{N}$ & $\%$ & $\mathbf{N}$ & $\%$ \\
\hline MULHERES & 66 & 72,5 & 22 & 33,3 & 18 & 27,3 & 26 & 39,4 \\
\hline HOMENS & 25 & 27,5 & 17 & 68 & 06 & 24 & 02 & 08 \\
\hline
\end{tabular}

Fonte: Autoria Própria.

Em relação ao RCV, notamos que usuários com alto RCV apresentaram média de pressão sistólica de $136 \mathrm{mmHg}$ para mulheres e $137 \mathrm{mmHg}$ para homens. A média do colesterol total e colesterol-HDL para as mulheres foi de $215 \mathrm{mg} / \mathrm{dL}$ e $54,6 \mathrm{mg} / \mathrm{dL}$ respectivamente, enquanto que os homens apresentaram média de colesterol total e colesterol-HDL de 190,7 mg/dL e 43,16 mg/dL (Tabela 2).

Tabela 2 - Distribuição das médias dos parâmetros analisados pela escala de Framingham.

\begin{tabular}{ccccc}
\hline SEXO & FAIXA ETÁRIA & $\begin{array}{c}\text { PRESSÃO } \\
\text { SISTÓLICA }\end{array}$ & HDL & $\begin{array}{c}\text { COLESTEROL } \\
\text { TOTAL }\end{array}$ \\
\hline FEMININO & $57( \pm 8,9)$ & $136( \pm 20)$ & $54( \pm 18)$ & $215( \pm 54)$ \\
MASCULINO & $59( \pm 7)$ & $137( \pm 21)$ & $43( \pm 17,7)$ & $190,7( \pm 42)$ \\
\hline
\end{tabular}

Fonte: Autoria Própria.

A maioria dos usuários hipertensos (75\%) utilizavam medicamentos anti-hipertensivos, enquanto que $25 \%$ não faziam uso de anti-hipertensivo, sendo que $65 \%$ eram mulheres e $35 \%$ homens. Em relação aos parâmetros diabetes, $65 \%$ dos usuários eram acometidos da doença, e desse total, $62,5 \%$ eram mulheres e $37,5 \%$ homens. Quanto ao parâmetro tabagismo, podemos observar que $96 \%$ dos usuários não eram tabagistas, enquanto que $4 \%$ eram tabagistas, desse total, 03 eram mulheres $(75,5 \%)$ e 01 era homem $(25,5 \%)$ (Tabela 3$)$.

Tabela 3- Distribuição dos usuários atendidos pelo programa Hiperdia na Unidade Básica de Saúde que não utilizam medicamentos anti-hipertensivos, são diabéticos e/ou tabagistas.

\begin{tabular}{ccccccc}
\hline SEXO & $\begin{array}{c}\text { NÃO USAM MEDICAMENTOS } \\
\text { ANTI-HEPERTENSIVO }\end{array}$ & \multicolumn{2}{c}{ DIABÉTICOS } & \multicolumn{2}{c}{ TABAGISTAS } \\
\hline & $\mathrm{N}$ & $\%$ & $\mathrm{~N}$ & $\%$ & $\mathrm{~N}$ & $\%$ \\
\hline GERAL & 23 & 100 & 32 & 100 & 04 & 100 \\
FEMININO & 15 & 65 & 20 & 62,5 & 03 & 75,5 \\
MASCULINO & 08 & 35 & 12 & 37,5 & 01 & 25,5 \\
\hline
\end{tabular}

Fonte: Autoria Própria.

\section{DISCUSSÃO}

$\mathrm{Na}$ análise dos prontuários constatou-se que a maior parte deles não apresentavam informações fundamentais para realização do cálculo do RCV, como idade do usuário (a qual não deve ser superior a 75 anos), uso de medicamento anti-hipertensivo, tabagismo, mensuração da pressão arterial e, resultado de exames de colesterol total e colesterol-HDL. É importante ressaltar que muitos prontuários continham solicitações de exames, que provavelmente não foram realizados, ou se foram, o profissional de saúde não 
anotou os resultados no prontuário, outro fato que devemos destacar diz respeito à validade dos exames anexados ou anotados no prontuário, alguns apresentavam validade defasada, de 10 anos atrás.

Nesse âmbito, o prontuário é um documento de suma importância, visto que além de reunir informações e dados relevantes sobre o paciente e/ou exames, também possibilita analisar a evolução do individuo assistido para que seja viável a definição da conduta profissional mais adequada para o caso (BRAGAS LZT, 2015).

Assim, o prontuário não preenchido corretamente pode implicar, indiretamente ou diretamente, em uma falta de comunicação entre integrantes da equipe multiprofissional, por conseguinte, na assistência do usuário (BRASIL, 2012).

Apesar da maior parcela dos prontuários não estarem em consonância com os parâmetros estabelecidos para o cálculo de RCV, os habilitados para o mesmo forneceram-nos informações suficientes para traçar um perfil dos usuários cadastrados no programa Hiperdia.

As falhas encontradas nas anotações dos prontuários serão de grande valia a equipe da unidade, pois através delas os profissionais terão a oportunidade de identificar sua origem, e trabalhar em uma resolutiva, aperfeiçoando assim a prestação de serviço aos usuários.

Observou-se que, a média da idade, tanto entre o sexo feminino quanto o masculino, fica entre 57,5 e 59 anos possuindo assim, relação direta com o surgimento da hipertensão, uma vez que, para Brandão et al. (2010) a pressão arterial possui aumento gradativo de acordo com o avanço da idade, acarretando assim, no surgimento dessa comorbidade

Constatou-se que havia um predomínio do sexo feminino entre os usuários cadastrados no programa e, que apesar de ser a maior parcela, as mulheres apresentaram um RCV duas vezes menor do que os homens de desenvolver alguma doença cardiovascular, isso ocorre, pois culturalmente são elas as que mais procuram o serviço de saúde pública, se comparadas aos homens.

Esse é um comportamento discutido por autores como Paula DJC (2014) e Rodrigues R, et al. (2010), como sendo resultante tanto da visão de que o homem é um ser invulnerável que reluta à prevenção e/ou tratamento de doenças, quanto ao fato de os serviços de saúde não estarem organizados para receber e captar essa demanda.

Um dos fatores que influenciam a adesão ao tratamento e a incidência de HAS é o sexo, sendo assim, a prevalência de hipertensão em mulheres pode se dar pelo fato da sua percepção em relação aos cuidados com a saúde, e por uma maior frequência desse público em serviços de saúde como atenção básica (ROCHA MLF et al, 2017).

Enquanto que os homens, de acordo com Leite et al. (2016), tendem a procurar os serviços de saúde principalmente em casos de emergência, ou seja, a prevenção de fatores de risco e a manutenção da saúde não são aspectos priorizados por grande parcela da população masculina, contribuindo para má qualidade de vida desse público.

Assim, pode-se evidenciar que a construção social da masculinidade está relacionada com a forma como os homens percebem o processo de cuidado consigo e o adoecer (GOMES R, 2007).

Após a análise dos dados e estratificação do RCV pelo aplicativo, notou-se que o mesmo forneceu dados relevantes sobre o perfil dos usuários da unidade, e sua incorporação ao atendimento dos usuários cadastrados no programa é de extrema importância, pois permite que os profissionais de saúde conduzam da melhor forma os cuidados adequados para cada indivíduo.

De acordo com Kukafka $R$, et al. (2015), aplicativos que geram informações de saúde e que as adaptam ao perfil do consumidor são importantes para influenciá-lo na direção de determinados estilos de vida saudáveis, o que demonstra a eficácia deste tipo de ferramenta, a qual pode possibilitar o monitoramento do perfil do usuário e, assim, fornecer uma assistência de qualidade. 


\section{CONSIDERAÇÕES FINAIS}

Por conseguinte, com a utilização do aplicativo para a estratificação de risco cardiovascular podemos verificar que a utilização de recursos tecnológicos fornece autonomia aos profissionais de saúde, uma vez que esses aplicativos são de fácil manuseio, economicamente viáveis, pois muitos estão disponíveis gratuitamente para diversos dispositivos móveis, como smartphones e tablets, o que otimiza o tempo dos profissionais, favorece a acessibilidade e melhora a qualidade do atendimento público.

\section{REFERÊNCIAS}

1. BERBEL NAN. Metodologia da problematização: Uma alternativa metodológica apropriada para o Ensino Superior. Seminário: Londrina, 1995; 16(2): 9-19.

2. BRAGAS LZT. A Importância da Qualidade Dos Registros De Enfermagem Para Gestão Em Saúde: Estudo Em Hospital Na Região Noroeste de RS. 2015. Trabalho de conclusão de curso (Especialização em gestão em saúde) - Universidade aberta do brasil, Porto Alegre, 2015.

3. BRANDÃO AA, MAGALHÃES MEC, ÁVILA A, et al. Conceituação, epidemiologia e prevenção primária. J. Bras. Nefrol, 2010; 32(1): 1-4.

4. BRASIL. Conselho federal de medicina. Resolução CFM n 1638/2002. Define prontuário médico e torna obrigatória a criação da comissão de revisão de prontuários nas instituições de saúde. Brasília: O conselho, 2002. Disponível em: http://www.portalmedico.org.br/resolucoes/cfm/2002/1638-2002.htm. Acesso em: 4 jul. 2019.

5. BRASIL. Ministério da Saúde. Portaria no. 2.436 de 21 de setembro de 2017. Brasília: Diário Oficial da República Federativa do Brasil, 2017.

6. BRASIL. Ministério da Saúde. Secretaria de Atenção à Saúde. Documento de diretrizes para o cuidado das pessoas com doenças crônicas nas redes de atenção à saúde e nas linhas de cuidado prioritárias. Brasília: Ministério da Saúde, 2013.

7. CARVALHO FC, ARRUDA PAC, BRUSTOLIN RD et al. Abordagem interdisciplinar sobre a prática da atividade física no grupo de hiperdia. VI Seminário Transdisciplinar da Saúde. Resumos. Mato Grosso: Centro Universitário de Várzea Grande, 2018.

8. CRUZ JVK, KIPPER LM. Desenvolvimento de um aplicativo para classificação de lesões por pressão na pele com o uso do design science research. Seminário de iniciação científica, 2018.

9. KUKAFKA R, JEONG IC, FINKELSTEIN J. Optimizing Decision Support for Tailored Health Behavior Change Applications. Studies in Health Technology and Informatics, 2015; (216): 108-112.

10. MALACHIAS MV, SOUZA WK, PLAVNIK FL et al. Sociedade Brasileira de Cardiologia. 7ª Diretriz Brasileira de hipertensão arterial. Arquivos Brasileiros de Cardiologia, 2016;107(3): 1-83.

11. PAULA DJC. Análise de custo e efetividade do tratamento de diabéticos adultos atendidos no centro Hiperdia de Juiz de Fora, Minas Gerais. [Dissertação]. Universidade Federal de Juiz de Fora. Programa de Pós-graduação de Juiz de Fora. Juiz de Fora, 2014; 119 p.

12. RAMOS S, MANENTI E, FRIENDRICH MAG et al. Entendendo as doenças cardiovasculares. 1. ed. Porto Alegre: Artmed, 2014; $104 \mathrm{p}$.

13. GOMES R. Sexualidade Masculina, Gênero e Saúde. Rio de Janeiro: Fiocruz; 2008.

14. ROCHA MLF et al. Adesão Ao Tratamento da Hipertensão Arterial Entre Usuários da Estratégia Saúde da Família Em Um Município Do Piauí. Rev. APS, 2017.

15. RODRIGUES R, POZZOBON A, HOERLLE J et al. Avaliação do perfil glicêmico de pacientes com diabetes mellitus tipo 2 com e sem administração de infusão de folhas de Averrhoa carambola. Scientia Medica, Porto Alegre, 2010; 20(2): 161-165.

16. ROTH GA, FOROUZANFAR MH, MORAN AE et al. Demographic and epidemiologic drivers of global cardiovascular mortality. New England Journal of Medicine, 2015; 372(14):1333-1341.

17. SCHMIDT L, BENETTI F, AIRES M. Avaliação do risco cardiovascular de pacientes diabéticos tipo 2. Revista de Pesquisa em Saúde, 2017; 18(2): 91-96.

18. SERAFIM GS, MORETTI M, SERAFIM AS et al. Avaliação do escore de risco de framingham em um ambulatório de cardiologia de criciúma - SC. Revista Inova Saúde, Criciúma, 2014; 3(2): 12. 